\title{
Ball convergence for combined three-step methods under generalized conditions in Banach space
}

\author{
Ioannis K. Argyros, Ramandeep Behl, \\ Daniel González and Sandile S. Motsa
}

\begin{abstract}
We give a local convergence analysis for an eighth-order convergent method in order to approximate a locally unique solution of nonlinear equation for Banach space valued operators. In contrast to the earlier studies using hypotheses up to the seventh Fréchet-derivative, we only use hypotheses on the first-order Fréchet-derivative and Lipschitz constants. Therefore, we not only expand the applicability of these methods but also provide the computable radius of convergence of these methods. Finally, numerical examples show that our results apply to solve those nonlinear equations but earlier results cannot be used.
\end{abstract}

Mathematics Subject Classification (2010): 65G99, 65H10, 47J25, 47J05, 65D10, 65 D99.

Keywords: Iterative method, local convergence, Banach space, Lipschitz constant, order of convergence.

\section{Introduction}

One of the most basic and important problems in Numerical Analysis concerns with approximating a locally unique solution $x^{*}$ of the equation of the form

$$
F(x)=0,
$$

where $F: \mathbb{D} \subset \mathbb{X} \rightarrow \mathbb{Y}$ is a Fréchet-differentiable operator, $\mathbb{X}, \mathbb{Y}$ are Banach spaces and $\mathbb{D}$ is a convex subset of $\mathbb{X}$. Let us also denote $L(\mathbb{X}, \mathbb{Y})$ as the space of bounded linear operators from $\mathbb{X}$ to $\mathbb{Y}$.

Approximating $x^{*}$ is very important, since numerous problems can be reduced to equation (1.1) using mathematical modeling [4, 7, 12, 9, 16, 21, 23, 24]. However, it is not always possible to find the solution $x^{*}$ in a closed form. Therefore, most of the 
methods are iterative to solve such type of problems. The convergence analysis of iterative methods is usually divided into two categories: semi-local and local convergence analysis. The semi-local convergence matter is, based on the information around an initial point, to give criteria ensuring the convergence of iteration procedures. A very important problem in the study of iterative procedures is the convergence domain. Therefore, it is very important to propose the radius of convergence of the iterative methods.

We study the local convergence of the three step eighth-order convergent method defined for each $n=0,12, \ldots$ by

$$
\begin{aligned}
y_{n} & =x_{n}-F^{\prime}\left(x_{n}\right)^{-1} F\left(x_{n}\right), \\
z_{n} & =\phi\left(x_{n}, F\left(x_{n}\right), F^{\prime}\left(x_{n}\right), F^{\prime}\left(y_{n}\right),\right. \\
x_{n+1} & =z_{n}-\beta A_{n}^{-1} F\left(z_{n}\right),
\end{aligned}
$$

where $x_{0} \in \mathbb{D}$ is an initial point, for $\alpha, \beta \in S, A_{n}=(\beta-\alpha) F^{\prime}\left(x_{n}\right)+\alpha F^{\prime}\left(y_{n}\right)$, $(S=$ $\mathbb{R}$ or $S=\mathbb{C}$ ) and the second sub step represents any iterative method, in which the order of convergence is at least $m=1,2,3, \ldots$ It was shown in [9] using Taylor series expansions when $\mathbb{X}=\mathbb{Y}=\mathbb{R}$ that method (1.2) is of order at least $2 m$, if $m<3$ and of order at least $m+3$, if $m \geq 3$ provided that $F$ is eighth times differentiable. The hypotheses on the derivatives of $F$ restrict the applicability of method (1.2). As a motivational example, define function $F$ on $\mathbb{X}=\mathbb{Y}=\mathbb{R}, D=\left[-\frac{3}{2}, \frac{1}{2}\right]$ by

$$
F(x)= \begin{cases}x^{3} \ln x^{2}+x^{5}-x^{4}, & x \neq 0 \\ 0, & x=0 .\end{cases}
$$

Then, we have that

$$
\begin{aligned}
& F^{\prime}(x)=3 x^{2} \ln x^{2}+5 x^{4}-4 x^{3}+2 x^{2}, \\
& F^{\prime \prime}(x)=6 x \ln x^{2}+20 x^{3}-12 x^{2}+10 x
\end{aligned}
$$

and

$$
F^{\prime \prime \prime}(x)=6 \ln x^{2}+60 x^{2}-24 x+22 .
$$

Then, obviously the third-order derivative of the involved function $F^{\prime \prime \prime}(x)$ is not bounded on $\mathbb{D}$. Notice that, in particular there is a plethora of iterative methods for approximating solutions of nonlinear equations $[2,1,3,4,5,7,8,6,10,11,12,13$, $14,15,16,17,18,19,20,21,22,23,24]$. These results show that initial guess should be close to the required root for the convergence of the corresponding methods. But, how close initial guess should be required for the convergence of the corresponding method? These local results give no information on the radius of the ball convergence for the corresponding method. We address this question for method (1.2) in the next section 2 .

In the present study, we expand the applicability of method (1.2) by using only hypotheses on the first-order derivative of function $F$ and generalized Lipschitz conditions. Moreover, we we will avoid to use Taylor series expansions and use Lipschitz parameters. In this way, there is no need to use the higher-order derivatives to show the convergence of the scheme (1.2). 
The rest of the paper is organized as follows: in section 2 contains the local convergence analysis of method (1.2). The numerical examples appear in the concluding Section 3.

\section{Local convergence}

The local convergence uses some scalars functions and parameters. Let $v, w_{0}, w, \bar{g}_{2}:[0,+\infty) \rightarrow[0,+\infty)$ be continuous, increasing functions with $w_{0}(0)=w(0)=0$ and $\alpha, \beta \in S$. Define parameter $r_{0}$ by

$$
r_{0}=\sup \left\{t \geq 0: w_{0}(t)<1\right\} .
$$

Moreover, define functions $g_{1}, h_{1}, p$ and $h_{p}$ on the interval $\left[0, r_{0}\right)$ by

$$
\begin{gathered}
g_{1}(t)=\frac{\int_{0}^{1} w((1-\theta) t) d \theta}{1-w_{0}(t)}, \\
h_{1}=g_{1}(t)-1, \\
p(t)=|\beta|^{-1}\left[|\beta-\alpha| w_{0}(t)+|\alpha| w_{0}\left(g_{1}(t) t\right)\right], \beta \neq 0,
\end{gathered}
$$

and

$$
h_{p}=p(t)-1 \text {. }
$$

We have by $(2.2)$ that $h_{1}(0)=h_{p}(0)=-1<0$ and $h_{1}(t) \rightarrow+\infty, h_{q}(t) \rightarrow+\infty$ as $t \rightarrow r_{0}^{-}$. Then, by the intermediate value theorem, we know that the functions $h_{1}$ and $h_{p}$ have zeros in the interval $\left(0, r_{0}\right)$. Denote by $r_{1}$ and $r_{p}$, respectively the smallest such zeros of the function $h_{1}$ and $h_{p}$. Furthermore, define functions $g_{2}$ and $h_{2}$ on the interval $\left(0, r_{0}\right)$ by

and

$$
g_{2}(t)=\bar{g}_{2}(t) t^{m-1}
$$

$$
h_{2}(t)=g_{2}(t)-1
$$

Suppose that

$$
\bar{g}_{2}(0)<1, \text { if } m=1
$$

and

$$
g_{2}(t) \rightarrow a \text { a number greater than one or }+\infty
$$

as $t \rightarrow \bar{r}_{0}^{-}$for some $\bar{r}_{0} \leq r_{0}$. Then, we have again by the intermediate value theorem that function $h_{2}$ has zeros in the interval $\left(0, \bar{r}_{0}\right)$. Denote by $r_{2}$ the smallest such zero. Notice that, if $m>1$ condition $(2.2)$ is not needed to show $h_{2}(0)<0$, since in this case $h_{2}(0)=g_{2}(0)-1=0-1=-1<0$. Finally, define functions $g_{3}$ and $h_{3}$ on the interval $\left[0, \bar{r}_{p}\right)$ by $\bar{r}_{p}=\min \left\{r_{p}, r_{2}\right\}$,

$$
g_{3}(t)=\left(1+\frac{\int_{0}^{1} v\left(\theta g_{2}(t) t\right) d \theta}{1-p(t)}\right) g_{2}(t),
$$

and

Suppose that

$$
h_{3}(t)=g_{3}(t)-1
$$

$$
(1+v(0)) \bar{g}_{2}(0)<1, \text { if } m=1,
$$


we get by $(2.4)$ that $h_{3}(0)=(1+v(0)) \bar{g}_{2}(0)-1<0$ and $h_{3}(t) \rightarrow+\infty$ or positive number as $t \rightarrow \bar{r}_{p}^{-}$. Denote by $r_{3}$ the smallest zero of function $h_{3}$ in the interval $\left(0, r_{p}\right)$. Define the radius of convergence $r$ by

$$
r=\min \left\{r_{1}, r_{3}\right\}
$$

Then, we have that for each $t \in[0, r)$

$$
0 \leq g_{i}(t)<1, i=1,2,3 .
$$

Let $U(z, \rho), \bar{U}(z, \rho)$, stand respectively for the open and closed balls in $\mathbb{X}$ with center $z \in \mathbb{X}$ and of radius $\rho>0$. Next, we present the local convergence analysis of method (1.2) using the preceding notations.

Theorem 2.1. Let $F: \mathbb{D} \subseteq \mathbb{X} \rightarrow \mathbb{Y}$ be a continuously Fréchet-differentiable operator. Let $v, w_{0}, w, \bar{g}_{2}:[0, \infty) \rightarrow[0, \infty)$ be increasing continuous functions with $w_{0}(0)=$ $w(0)=0$ and let $r_{0} \in[0, \infty), \alpha \in S, \beta \in S-\{0\}, m \geq 1$ and $r_{0}$ be defined by $(2.1)$ so that (2.1) and (2.2) are satisfied. Suppose that there exists $x^{*} \in \mathbb{D}$ such that for each $x \in \mathbb{D}$ parameter $r_{0}$ be defined by $(2.1)$.

$$
F\left(x^{*}\right)=0, \quad F^{\prime}\left(x^{*}\right)^{-1} \in L(\mathbb{Y}, \mathbb{X})
$$

and

$$
\| F^{\prime}\left(x^{*}\right)^{-1}\left(F^{\prime}(x)-F^{\prime}\left(x^{*}\right) \| \leq w_{0}\left(\left\|x-x^{*}\right\|\right) .\right.
$$

Moreover, suppose that for each $x, y \in \mathbb{D}_{0}:=\mathbb{D} \cap U\left(x^{*}, r_{0}\right)$

$$
\begin{gathered}
\left\|F^{\prime}\left(x^{*}\right)^{-1}\left(F^{\prime}(x)-F^{\prime}(y)\right)\right\| \leq w(\|x-y\|), \\
\left\|F^{\prime}\left(x^{*}\right)^{-1} F^{\prime}(x)\right\| \leq v\left(\left\|x-x^{*}\right\|\right), \\
\left\|\phi\left(x, F(x), F^{\prime}(x), F^{\prime}(y)\right)\right\| \leq \bar{g}_{2}\left(\left\|x-x^{*}\right\|\right)\left\|x-x^{*}\right\|^{m}
\end{gathered}
$$

and

$$
\bar{U}\left(x^{*}, r\right) \subseteq \mathbb{D},
$$

where the radius of convergence $r$ is defined by (2.3). Then, sequence $\left\{x_{n}\right\}$ generated for $x_{0} \in U\left(x^{*}, r\right)-\left\{x^{*}\right\}$ by method (1.2) is well defined, remains in $U\left(x^{*}, r\right)$ for each $n=0,1,2, \ldots$ and converges to $x^{*}$. Moreover, the following estimates hold

$$
\begin{gathered}
\left\|y_{n}-x^{*}\right\| \leq g_{1}\left(\left\|x_{n}-x^{*}\right\|\right)\left\|x_{n}-x^{*}\right\| \leq\left\|x_{n}-x^{*}\right\|<r, \\
\left\|z_{n}-x^{*}\right\| \leq g_{2}\left(\left\|x_{n}-x^{*}\right\|\right)\left\|x_{n}-x^{*}\right\| \leq\left\|x_{n}-x^{*}\right\|
\end{gathered}
$$

and

$$
\left\|x_{n+1}-x^{*}\right\| \leq g_{3}\left(\left\|x_{n}-x^{*}\right\|\right)\left\|x_{n}-x^{*}\right\| \leq\left\|x_{n}-x^{*}\right\|,
$$

where the functions $g_{i}, i=1,2,3$ are defined above the Theorem. Furthermore, if

$$
\int_{0}^{1} w_{0}(\theta R) d \theta<1, \text { for } R \geq r,
$$

then the point $x^{*}$ is the only solution of equation $F(x)=0$ in $\mathbb{D}_{1}:=\mathbb{D} \cap \bar{U}\left(x^{*}, R\right)$. 
Proof. We shall show using mathematical induction that the sequences $\left\{x_{n}\right\}$ is well defined in $U\left(x^{*}, r\right)$ and converges to $x^{*}$. By the hypothesis $x_{0} \in U\left(x^{*}, r\right)-\left\{x^{*}\right\}$, (2.1), (2.3) and (2.10), we have that

$$
\left\|F^{\prime}\left(x^{*}\right)^{-1}\left(F^{\prime}\left(x_{0}\right)-F^{\prime}\left(x^{*}\right)\right)\right\| \leq w_{0}\left(\left\|x_{0}-x^{*}\right\|\right)<w_{0}(r)<1 .
$$

In view of (2.17) and the Banach Lemma on invertible operators $[4,7]$ that $F^{\prime}\left(x_{0}\right)^{-1} \in$ $L(\mathbb{Y}, \mathbb{X}), y_{0}$ is well defined by the first two sub steps of method (1.2) and

$$
\left\|F^{\prime}\left(x_{0}\right)^{-1} F^{\prime}\left(x^{*}\right)\right\| \leq \frac{1}{1-w_{0}\left(\left\|x_{0}-x^{*}\right\|\right)} .
$$

We get by (2.1), (2.5), (2.6) (for $i=1),(2.7)$ and (2.18) that

$$
\begin{aligned}
\left\|y_{0}-x^{*}\right\| & =\left\|\left(x_{0}-x^{*}-F^{\prime}\left(x_{0}\right)^{-1} F\left(x_{0}\right)\right)\right\| \\
& \leq\left\|F^{\prime}\left(x_{0}\right)^{-1} F\left(x^{*}\right)\right\| \| \int_{0}^{1} F^{\prime}\left(x^{*}\right)^{-1}\left(F^{\prime}\left(x^{*}+\theta\left(x_{0}-x^{*}\right)\right)\right. \\
& \left.-F^{\prime}\left(x_{0}\right)\right)\left(x_{0}-x^{*}\right) d \theta \| \\
& \leq \frac{\int_{0}^{1} w\left((1-\theta)\left\|x_{0}-x^{*}\right\|\right) d \theta\left\|x_{0}-x^{*}\right\|}{1-w_{0}\left(\left\|x_{0}-x^{*}\right\|\right)} \\
& \leq g_{1}\left(\left\|x_{0}-x^{*}\right\|\right)\left\|x_{0}-x^{*}\right\| \leq\left\|x_{0}-x^{*}\right\|<r,
\end{aligned}
$$

which implies (2.13) for $n=0$ and $y_{0} \in U\left(x^{*}, r\right)$. By (2.5), (2.6) (for $i=2$ ) and (2.11), we obtain in turn that

$$
\begin{aligned}
\left\|z_{0}-x^{*}\right\| & =\left\|\phi\left(x_{0}, F\left(x_{0}\right), F^{\prime}\left(x_{0}\right), F^{\prime}\left(y_{0}\right)\right)\right\| \\
& \leq \bar{g}_{2}\left(\left\|x_{0}-x^{*}\right\|\right)\left\|x_{0}-x^{*}\right\|^{m} \\
& =g_{2}\left(\left\|x_{0}-x^{*}\right\|\right)\left\|x_{0}-x^{*}\right\| \leq\left\|x_{0}-x^{*}\right\|<r,
\end{aligned}
$$

which shows (2.14) for $n=0$ and $z_{0} \in U\left(x^{*}, r\right)$. We must show that $x_{1}$ exists. Using $(2.1),(2.5)$ and $(2.8)$, we obtain in turn that

$$
\begin{aligned}
\|\left(\beta F^{\prime}\left(x^{*}\right)\right)^{-1}[(\beta- & \left.\alpha)\left(F^{\prime}\left(x_{0}\right)-F^{\prime}\left(x^{*}\right)\right)+\alpha\left(F^{\prime}\left(y_{0}\right)-F^{\prime}\left(x^{*}\right)\right)\right] \| \\
& \leq|\beta|^{-1}\left[|\beta-\alpha| w_{0}\left(\left\|x_{0}-x^{*}\right\|\right)+|\alpha| w_{0}\left(\left\|y_{0}-x^{*}\right\|\right)\right] \\
& \leq|\beta|^{-1}\left[|\beta-\alpha| w_{0}\left(\left\|x_{0}-x^{*}\right\|\right)+|\alpha| w_{0}\left(g_{1}\left(\left\|x_{0}-x^{*}\right\|\right)\left\|x_{0}-x^{*}\right\|\right)\right] \\
& =p\left(\left\|x_{0}-x^{*}\right\|\right) \leq p(r)<1,
\end{aligned}
$$

SO

$$
\left\|\left((\beta-\alpha) F^{\prime}\left(x_{0}\right)+\alpha F^{\prime}\left(y_{0}\right)\right)^{-1} F^{\prime}\left(x^{*}\right)\right\| \leq \frac{1}{1-p\left(\left\|x_{0}-x^{*}\right\|\right)} .
$$


Then, from the last sub step of method (2.1), (2.5), (2.6) (for $i=3$ ), (2.10), (2.19), (2.20) and (2.21), we get in turn that

$$
\begin{aligned}
\left\|x_{1}-x^{*}\right\| & =\left\|z_{0}-x^{*}\right\|+|\beta| \int_{0}^{1} v\left(\theta\left\|z_{0}-x^{*}\right\|\right) d \theta\left\|x_{0}-x^{*}\right\| \\
& \leq\left(1+\frac{|\beta| \int_{0}^{1} v\left(\theta g_{2}\left(\left\|x_{0}-x^{*}\right\|\right)\right) d \theta}{|\beta|\left(1-p\left(\left\|x_{0}-x^{*}\right\|\right)\right)}\right) g_{2}\left(\left\|x_{0}-x^{*}\right\|\right)\left\|x_{0}-x^{*}\right\| \\
& =g_{3}\left(\left\|x_{0}-x^{*}\right\|\right)\left\|x_{0}-x^{*}\right\| \leq\left\|x_{0}-x^{*}\right\|<r,
\end{aligned}
$$

which shows (2.15) and $x_{1} \in U\left(x^{*}, r\right)$. By simply replacing $x_{0}, y_{0}, x_{1}$ by $x_{k}, y_{k}$, $x_{k+1}$ in the preceding estimates we arrive at (2.15) and (2.16). Then, in view of the estimates

$$
\left\|x_{k+1}-x^{*}\right\| \leq c\left\|x_{k}-x^{*}\right\|<r, c=g_{2}\left(\left\|x_{0}-x^{*}\right\|\right) \in[0,1),
$$

we deduce that $\lim _{k \rightarrow \infty} x_{k}=x^{*}$ and $x_{k+1} \in U\left(x^{*}, r\right)$. Finally, to show the uniqueness part, let $y^{*} \in D_{1}$ with $F\left(y^{*}\right)=0$. Define $Q=\int_{0}^{1} F^{\prime}\left(x^{*}+\theta\left(x^{*}-y^{*}\right)\right) d \theta$. Using (2.5) and (2.12), we get that

$$
\begin{aligned}
\left\|F^{\prime}\left(x^{*}\right)^{-1}\left(Q-F^{\prime}\left(x^{*}\right)\right)\right\| & \leq \| \int_{0}^{1} w_{0}\left(\theta\left\|y^{*}-x^{*}\right\|\right) d \theta \\
& \leq \int_{0}^{1} w_{0}(\theta R) d \theta<1 .
\end{aligned}
$$

It follows from (2.25) that $Q$ is invertible. Then, in view of the identity

$$
0=F\left(x^{*}\right)-F\left(y^{*}\right)=Q\left(x^{*}-y^{*}\right),
$$

we conclude that $x^{*}=y^{*}$.

Remark 2.2. (a) It follows from (2.10) that condition (2.12) can be dropped and be replaced by

$$
v(t)=1+w_{0}(t) \text { or } v(t)=1+w_{0}\left(r_{0}\right)
$$

since,

$$
\begin{aligned}
\left\|F^{\prime}\left(x^{*}\right)^{-1}\left[\left(F^{\prime}(x)-F^{\prime}\left(x^{*}\right)\right)+F^{\prime}\left(x^{*}\right)\right]\right\| & =1+\left\|F^{\prime}\left(x^{*}\right)^{-1}\left(F^{\prime}(x)-F^{\prime}\left(x^{*}\right)\right)\right\| \\
& \leq 1+w_{0}\left(\left\|x-x^{*}\right\|\right) \\
& =1+w_{0}(t) \text { for }\left\|x-x^{*}\right\| \leq r_{0} .
\end{aligned}
$$

(b) If the function $w_{0}$ is strictly increasing, then we can choose

$$
r_{0}=w_{0}^{-1}(1)
$$

instead of (2.1).

(c) If $w_{0}, w, v$ are constants functions (the proof of Theorem 2.1 goes through too in this case), then

and

$$
r_{1}=\frac{2}{2 w_{0}+w}
$$

$$
r \leq r_{1}
$$


Therefore, the radius of convergence $r$ can be larger than the radius of convergence $r_{1}$ for Newton's method

$$
x_{n+1}=x_{n}-F^{\prime}\left(x_{n}\right)^{-1} F\left(x_{n}\right) .
$$

Notice also that the earlier radius of convergence given independently by Rheindoldt $[22]$ and Traub [24] is

and by Argyros $[4,7]$

$$
r_{T R}=\frac{2}{3 w_{1}}
$$

$$
r_{A}=\frac{2}{2 w_{0}+w_{1}}
$$

where $w_{1}$ is the Lipschitz constant for $(2.6)$ on $D$. But, we have

$$
w \leq w_{1}, w_{0} \leq w_{1}
$$

so

$$
r_{T R} \leq r_{A} \leq r_{1}
$$

and

$$
\frac{r_{T R}}{r_{A}} \rightarrow \frac{1}{3} \quad \text { as } \quad \frac{w_{0}}{w} \rightarrow 0
$$

The radius of convergence $q$ used in [9] is smaller than the radius $r_{D S}$ given by Dennis and Schabel [4]

$$
q<r_{S D}=\frac{1}{2 w_{1}}<r_{T R}
$$

However, $q$ can not be computed using the Lipschitz constants.

(d) The results obtained here can be used for operators $F$ satisfying the autonomous differential equation $[4,7]$ of the form

$$
F^{\prime}(x)=P(F(x))
$$

where $P$ is a known continuous operator. Since $F^{\prime}\left(x^{*}\right)=P\left(F\left(x^{*}\right)\right)=P(0)$, we can apply the results without actually knowing the solution $x^{*}$. Let as an example $F(x)=e^{x}-1$. Then, we can choose $P(x)=x+1$.

(e) Let us show how to choose functions $\phi, \overline{g_{2}}, g_{2}$ and $m$. In addition, we assume that $\mathbb{X}=\mathbb{Y}=\mathbb{R}$. Define function $\phi$ on $\mathbb{R}^{4}$ by

$$
\phi\left(x_{n}, F\left(x_{n}\right), F^{\prime}\left(x_{n}\right), F^{\prime}\left(y_{n}\right)\right)=y_{n}-F^{\prime}\left(y_{n}\right)^{-1} F\left(y_{n}\right) .
$$

Then, we can choose

$$
g_{2}(t)=\frac{\int_{0}^{1} w\left((1-\theta) g_{1}(t) t\right) d \theta g_{1}(t)}{1-w_{0}\left(g_{1}(t) t\right)}
$$

If $w_{0}, w, v$ are given in particular by $w_{0}(t)=L_{0} t, w(t)=L t$ and $v(t)=<$ for some $L>0, L>0$ and $M \geq 1$, then we have that

$$
\begin{aligned}
\bar{g}_{2}(t) & =\frac{\frac{L^{2}}{8\left(1-L_{0} t\right)^{2}}}{1-\frac{L_{0} L t^{2}}{2\left(1-L_{0} t\right)}} \\
g_{2}(t) & =\bar{g}_{2}(t) t^{3} \text { and } m=4 .
\end{aligned}
$$


(f) If $\beta=0$, we can obtain the results for the two-step method

$$
\begin{aligned}
y_{n} & =x_{n}-F^{\prime}\left(x_{n}\right)^{-1} F\left(x_{n}\right), \\
x_{n+1} & =\phi\left(x_{n}, F\left(x_{n}\right), F^{\prime}\left(x_{n}\right), F^{\prime}\left(y_{n}\right)\right)
\end{aligned}
$$

by setting $z_{n}=x_{n+1}$ in Theorem 2.1 .

\section{Numerical examples and applications}

In this section, we shall demonstrate the theoretical results which we have proposed in the section 2. Therefore, we consider four numerical examples in this section, which are defined as follows:

Example 3.1. Let $X=Y=C[0,1]$ and consider the nonlinear integral equation of the mixed Hammerstein-type $[13,16]$, defined by

$$
x(s)=\int_{0}^{1} G(s, t)\left(x(t)^{\frac{3}{2}}+\frac{x(t)^{2}}{2}\right) d t
$$

where the kernel $G$ is the Green's function defined on the interval $[0,1] \times[0,1]$ by

$$
F(s, t)=\left\{\begin{array}{l}
(1-s) t, t \leq s, \\
s(1-t), s \leq t .
\end{array}\right.
$$

The solution $x^{*}(s)=0$ is the same as the solution of equation (1.1), where $F: \subseteq$ $C[0,1] \rightarrow C[0,1]$ defined by

$$
F(x)(s)=x(s)-\int_{0}^{t} G(s, t)\left(x(t)^{\frac{3}{2}}+\frac{x(t)^{2}}{2}\right) d t .
$$

Notice that

$$
\left\|\int_{0}^{t} G(s, t) d t\right\| \leq \frac{1}{8}
$$

Then, we have that

$$
F^{\prime}(x) y(s)=y(s)-\int_{0}^{t} G(s, t)\left(\frac{3}{2} x(t)^{\frac{1}{2}}+x(t)\right) d t,
$$

so since $F^{\prime}\left(x^{*}(s)\right)=I$,

$$
\left\|F^{\prime}\left(x^{*}\right)^{-1}\left(F^{\prime}(x)-F^{\prime}(y)\right)\right\| \leq \frac{1}{8}\left(\frac{3}{2}\|x-y\|^{\frac{1}{2}}+\|x-y\|\right) .
$$

Therefore, we can choose

$$
w_{0}(t)=w(t)=\frac{1}{8}\left(\frac{3}{2} t^{\frac{1}{2}}+t\right)
$$

and by Remark 2.2(a)

$$
v(t)=1+w_{0}(t) .
$$

The results in $[16,9]$ can not be used to solve this problem, since $F^{\prime}$ is not Lipschitz. However, our results can apply. 
Example 3.2. Suppose that the motion of an object in three dimensions is governed by system of differential equations

$$
\begin{aligned}
& f_{1}^{\prime}(x)-f_{1}(x)-1=0 \\
& f_{2}^{\prime}(y)-(e-1) y-1=0 \\
& f_{3}^{\prime}(z)-1=0
\end{aligned}
$$

with $x, y, z \in \Omega$ for $f_{1}(0)=f_{2}(0)=f_{3}(0)=0$. then, the solution of the system is given for $w=(x, y, z)^{T}$ by function $F:=\left(f_{1}, f_{2}, f_{3}\right): \Omega \rightarrow \mathbb{R}^{3}$ defined by

$$
F(v)=\left(e^{x}-1, \frac{e-1}{2} y^{2}+y, z\right)^{T} .
$$

Then the Fréchet-derivative is given by

$$
F^{\prime}(v)=\left[\begin{array}{ccc}
e^{x} & 0 & 0 \\
0 & (e-1) y+1 & 0 \\
0 & 0 & 1
\end{array}\right] .
$$

Then, we have that $w_{0}(t)=L_{0} t, w(t)=L t, w_{1}(t)=L_{1} t, w_{0}=L_{0}, w_{1}=L_{1}$ and $v(t)=M$, where $L_{0}=e-1<L=e^{\frac{1}{L_{0}}}=1.789572397, L_{1}=e$ and $M=e^{\frac{1}{L_{0}}}=$ 1.7896. Then, we get

$$
r=0.0039782 .
$$

Example 3.3. Let $A_{1}=A_{2}=C[0,1]$, be the space of continuous functions defined on the interval $[0,1]$ and be equipped with max norm. Let $\Omega=\bar{U}(0,1)$ and $B(x)=$ $F^{\prime \prime}(x)$ for each $x \in \Omega$. Define $F$ on $\Omega$

$$
F(\varphi)(x)=\phi(x)-5 \int_{0}^{1} x \theta \varphi(\theta)^{3} d \theta .
$$

We have that

$$
F^{\prime}(\varphi(\xi))(x)=\xi(x)-15 \int_{0}^{1} x \theta \varphi(\theta)^{2} \xi(\theta) d \theta, \text { for each } \xi \in \Omega .
$$

Then, we have that $x^{*}=0, L_{0}=7.5, L_{1}=L=15$ and $M=2$. Using method (1.2) for $w_{0}(t)=L_{0} t, v(t)=2=M, w(t)=L t, w_{1}=L$ and $w_{0}=L_{0}$, we get

$$
r=0.0013404 \text {. }
$$

Example 3.4. Returning back to the motivation example at the introduction on this paper, we have $L=L_{0}=96.662907$ and $M=2$. Using method (1.2) for $w_{0}(t)=$ $L_{0} t, v(t)=2=M, w(t)=L t, w_{1}(t)=L$ and $w_{0}=L_{0}$, we can choose

$$
r=0.00085 \text {. }
$$

\section{References}

[1] Amat, S., Busquier, S., Plaza, S., Dynamics of the King and Jarratt iterations, Aequationes Math., 69(2005), no. 3, 212-223.

[2] Amat, S., Busquier, S., Plaza, S., Gutiérrez, J.M., Geometric constructions of iterative functions to solve nonlinear equations, J. Comput. Appl. Math., 157(2003), 197-205. 
[3] Amat, S., Hernández, M.A., Romero, N., A modified Chebyshev's iterative method with at least sixth order of convergence, Appl. Math. Comput., 206(2008), no. 1, 164-174.

[4] Argyros, I.K., Convergence and Application of Newton-type Iterations, Springer, 2008.

[5] Argyros, I.K., George, S., Ball comparison for three optimal eight order methods under weak conditions, Stud. Univ. Babeş-Bolyai Math., 64(2019), no. 3, 421-431.

[6] Argyros, I.A., George, S., Local convergence of some higher-order Newton-like method with frozen derivative, SeMa, DOI: 10.1007/s40324-015-00398-8.

[7] Argyros, I.K., Hilout, S., Computational Methods in Nonlinear Analysis, World Scientific Publ. Comp. New Jersey, 2013.

[8] Argyros, I.K., Magreñán, Á.A., Ball convergence theorems and the convergence planes of an iterative methods for nonlinear equations, SeMA, 71(2015), no. 1, 39-55.

[9] Chen, S.P., Qian, Y.H., A family of combined iterative methods for solving nonlinear equations, Appl. Math. Comput. (to appear).

[10] Cordero, A., Torregrosa, J.R., Variants of Newton's method using fifth-order quadrature formulas, Appl. Math. Comput., 190(2007), 686-698.

[11] Cordero, A., Torregrosa, J.R., Variants of Newton's method for functions of several variables, Appl. Math. Comput., 183(2006), 199-208.

[12] Cordero, A., Torregrosa, J.R., Vassileva, M.P., Increasing the order of convergence of iterative schemes for solving nonlinear system, J. Comput. Appl. Math., 252(2012), 8694.

[13] Ezquerro, J.A., Hernández, M.A., New iterations of R-order four with reduced computational cost, BIT Numer. Math., 49(2009), 325-342.

[14] Ezquerro, J.A., Hernández, M.A., A uniparametric Halley type iteration with free second derivative, Int. J. Pure and Appl. Math., 6(2003), no. 1, 99-110.

[15] Gutiérrez, J.M., Hernández, M.A., Recurrence relations for the super-Halley method, Comput. Math. Appl., 36(1998), 1-8.

[16] Hernández, M.A., Martinez, E., On the semilocal convergence of a three steps Newtontype process under mild convergence conditions, Numer. Algor., 70(2015), 377-392.

[17] Kansal, M., Argyros, I.K., Kanwar, V., Ball convergence of a stable forth-order family for solving nonlinear systems under weak conditions, Stud. Univ. Babeş-Bolyai Math., 62(2017), no. 1, 127-135.

[18] Kou, J., A third-order modification of Newton method for systems of nonlinear equations, Appl. Math. Comput., 191(2007), 117-121.

[19] Montazeri, H., Soleymani, F., Shateyi, S., Motsa, S.S., On a new method for computing the numerical solution of systems of nonlinear equations, J. Appl. Math., 2012, Article ID 751975 .

[20] Petkovic, M.S., Neta, B., Petkovic, L., Džunič, J., Multipoint Methods for Solving Nonlinear Equations, Elsevier, 2013.

[21] Potra, F.A., Pták, V., Nondiscrete Introduction and Iterative Process, Research Notes in Mathematics, 103(1984), Pitman, Boston, MA.

[22] Rheinboldt, W.C., An Adaptive Continuation Process for Solving Systems of Nonlinear Equations, Polish Academy of Science, Banach Ctr. Publ., 3(1978), 129-142.

[23] Sharma, J.R., Ghua, R.K., Sharma, R., An efficient fourth-order weighted-Newton method for system of nonlinear equations, Numer. Algor., 62(2013), 307-323. 
[24] Traub, J.F., Iterative methods for the solution of equations, Prentice-Hall Series in Automatic Computation, Englewood Cliffs, N.J. (1964).

Ioannis K. Argyros

Cameron University,

Department of Mathematics Sciences,

Lawton, OK 73505, USA

e-mail: ioannisa@cameron.edu

Ramandeep Behl

Department of Mathematics,

Faculty of Science,

King Abdulaziz University,

Jeddah 21589, Saudi Arabia

e-mail: ramanbeh187@yahoo.in

Daniel González

Universidad de Las Américas,

Escuela de Ciencias Físicas y Matemáticas,

Quito, 170125, Ecuador

e-mail: daniel.gonzalez.sanchez@udla.edu.ec

Sandile S. Motsa

University of KwaZulu-Natal,

School of Mathematics, Statistics and Computer Sciences,

Private Bag X01, Scottsville 3209,

Pietermaritzburg, South Africa

e-mail: sandilemotsa@gmail.com 